\title{
Aprendizaje Basado en el Cerebro
}

\section{Brain Based Learning}

María de los Angeles Saavedra, PhD *

\section{Resumen}

El artículo que se presenta es un intento por compartir con psicólogos y educadores la integración que proponen algunos autores entre el conocimiento sobre el cerebro y el aprendizaje en el aula, un problema arduo para la educación. En las tres o cuatro últimas décadas debido principalmente a los adelantos de las técnicas neurofisiológicas se ha avanzado en forma sustancial en el conocimiento del funcionamiento del sistema nervioso y cerebral, lo que ha permitido adelantar hipótesis relacionadas con la situacion óptima para que el cerebro y el sistema neuroendocrino efectúe su mejor aprendizaje. Se revisan algunos antecedentes y el desarrollo de las ideas en torno al aprendizaje basado en el cerebro.

Los aportes principales se encuentran en el desarrollo del concepto de plasticidad cerebral, aprendizaje con significado, el efecto de la experiencia temprana enriquecida, el efecto de la nutrición y los ritmos temporales naturales del ser vivo que influyen sobre la atención. El artículo finaliza con un resumen de los principios más importantes sustentados por Caine, Caine y Crowell (1999) sobre aprendizaje basado en el cerebro, en el que destaca el principio de unicidad y diversidad de la organización cerebral de los seres vivientes.

Palabras Claves: Plasticidad y Aprendizaje

\section{Abstract}

The purpose of the present article is to share with psychologists and educators the integration proposed by several authors between what is known about the brain and the process of learning as it goes on in schools. Due to the developement of neurophysiological techniques during the recent decades, particularly imaging, an important advance in the knowledge of the functioning of the neuroendocrine system and brain mechanisms has been evidenced. This has permitted the posing of certain principles as to the cerebrum's optimum state for learning. The background and development of the ideas related to brain based learning are reviewed.

* Académico, Departamento de Psicología, Universidad de Chile email: mignon@entelchile.net 
The main contributions can be found in the development of the concept of cerebral plasticity, significant learning, the effect of enriched early environment, the effect of nutrition and water, and the innate temporal rythms of living organisms which are influential on attention and focusing. The article ends with a summary of the most important principles suggested by Caine, Caine and Crowell (1999) on brain based learning.

Key Words: Learning Plasticity-Brain

Una de las aplicaciones importantes que ha tenido el desarrollo de las Neurociencias en las últimas dos décadas ha sido en el campo de la Educación. En el presente trabajo se intenta compartir con el ámbito de la educación y la psicología la relevancia que adquiere el conocimiento del funcionamiento del cerebro en los procesos de aprendizaje. No está lejana la época en que las disciplinas de la Neurobiología y las del área educacional marchaban en forma paralela e independiente la una de la otra. Durante largo tiempo la educación y la psicología fisiológica se mantuvieron separadas, sin aportarse mutuamente en lo conceptual, prácticamente sin interesarse la una por la otra. Esto ocurrió, en parte, por el hecho de que la mayor parte de la investigación en psicología fisiológica estaba más preocupada por los mecanismos cerebrales de funcionamiento en ratas de laboratorio que aprendían un recorrido en un laberinto, que con el aprendizaje de los niños en las aulas. La relación entre la Educación y la Psicología en lo biológico estaba dirigida hacia su relación con problemas clínicos de aprendizaje (como las dislexias) en lugar de los principios del aprendizaje por mecanismos que se realizan en un cerebro normal debido a un funcionamiento neuroendocrino normal. Una excepción fue el área de la Psicología Experimental del Condicionamiento y los principios de refuerzo, aunque como siempre mirado solo desde la (mala o conflictiva) conducta de un Sujeto que aprende con- tenidos, sin considerar mayormente el cerebro y el sistema neuroendocrino del sujeto que aprende. El aporte de los trabajos en condicionamiento fue principalmente metodológico y estuvo lejos -con excepción de los trabajos de Skinner que si lo intentaron-de solucionar el problema para un aprendizaje más eficiente .

Ahora ha llegado el momento de hacer énfasis tanto en las áreas de la psicología clínica como educacional de algunos aspectos de la fisiología cerebral cuyo conocimiento puede ser un aporte útil para una mejor comprensión de los procesos y mecanismos propios del aprender. Para la clínica, porque en último término es el cerebro, que al regular los procesos cognitivos y emocionales, hará posible que el paciente aprenda a tener una mirada diferente de sus padecimientos. Para la educación, porque tambien dependerá del estado óptimo en que se encuentre el cerebro para que se establezcan las nuevas conexiones que permitirán la adquisición de estrategias necesarias para la sobrevivencia . Es así, como Jensen (1996) habla de paradigmas cambiantes: de la Psicología a la Biología. De un enfoque psicológico conductista Skinneriano de los años 5060 , se ha pasado a uno psicobiológico, personificados por neurocientistas tales como Gazzaniga, Damasio, Restak, y Edelman, por mencionar unos pocos, el que puede resumirse en la orientación Psicobiológica Cognitiva del fin de milenio. 


\section{Antecedentes}

Fueron los esposos Caine y Caine (1994) los que en su libro "Haciendo conexiones: la enseñanza y el Cerebro Humano", expresaron con toda claridad la necesidad de un conocimiento actualizado de lo que ocurre durante el aprendizaje en el cerebro, que al fin y al cabo, es el órgano que por medio de la conectividad neuronal hace posible el aprendizaje .

Podría decirse que el resultado de investigaciones que produjeron el primer impacto directo sobre la educación fue, el de hace ya más de 20 años, cuando se describieron algunas funciones de los hemisferios cerebrales izquierdo y derecho, producto de las evaluaciones neuropsicológicas efectuadas en pacientes comisurotomizados - pacientes epilépticos intratables con cerebro dividido (Sperry, 1974). Estos resultados llevaron a la caracterización de un hemisferio derecho globalista u holista e intuitivo y un hemisferio izquierdo analítico, secuencial y detallista. En Educación repercutió en la forma de lo que se denominó "estilos de aprendiza$\mathrm{je}^{\prime \prime}$, término con el que el ámbito educacional está plenamente familiarizado. Posiblemente estos hallazgos provenientes de los estudios de lateralización hemisférica fueron sobrevalorados, porque no hay que olvidar que la descripción del funcionamiento de cada hemisferio se estaba haciendo a partir de las observaciones realizadas en pacientes crónicos con cerebros divididos. Lo normal es una función cerebral con cuerpo calloso intacto que permite acceder a la información prácticamente simultánea a ambos hemisferios. El hemisferio izquierdo (en los diestros) se hace cargo de la comprensión y comunicación verbal, el análisis secuencial y la planificación; el hemisferio derecho del reconocimiento y la expresión de las emociones, el reconocimiento de patrones musicales y posee reconocimiento de lenguaje no verbal, por una parte y conciencia de sí mismo, algún lenguaje y capacidad de aprendizaje, por otra. Con el énfasis actual sobre la importancia que adquieren las emociones en el aprendizaje (Goleman, 1995 se añade un elemento más que ayuda a entender la mayor facilidad de adquisición de hechos "significativos" en el proceso enseñanza-aprendizaje, lo cual conduce a la mayor comprensión de los mismos.

Para el campo de la educación no es novedad que la comprensión da lugar a aprendizajes más profundos y de mas largo alcance, que los aprendizajes de "contenidos" pues aunque estos tambien pueden realizarse (de memoria), y se continúan llevando a cabo en la educación pasiva, requieren de mucha práctica para que no sean de corta duración y no se prestan al aprendizaje de reglas, ni a los procesos de generalización requeridos para el aprendizaje de "aprender a aprender". Esta idea está muy bien formulada en el libro de los Caine ya citado ( 1994) .

La idea de los Caine es que mientras más conexiones entre neuronas tenga el cerebro que aprende, lo que se logra con una rica experiencia, habrá mayor comprensión del nuevo material a ser aprendido, pues la nueva información puede relacionarse (el tradicional concepto de "asociación") con la ya habida, y efectuar conexiones con contenidos existentes. Como consecuencia, el educador debiera enseñar su materia relacionándola con lo que el niño trae a la situación de aprendizaje. La idea ha sido puesta en práctica en la reforma educacional; $y$ mirado desde la perspectiva neurobiológica indica que el niño no llega a la situación con un cerebro parecido a una "tabula rasa", sino que trae una serie de experiencias provenientes de su situación familiar y de su particular socialización, los que ya han permitido el establecimiento de numerosas conexiones neuronales en el cerebro, debido a los aprendizajes ya efectuados. Al tomar esto en cuenta el edu- 
cador, facilitará extraordinariamente el aprendizaje del educando. Todo el movimiento generado por el modelo MITA (Weber, 1999) -enfoque educativo basado en las Inteligencias Múltiples- es una forma de aplicación de estos principios.

\section{Desarrollo de las ideas relacionadas con el aprendizaje basado en el cerebro}

No hay duda que uno de los problemas fundamentales de cualquier reforma -y lo será en la reforma educacional- es cómo hacer que importantes nuevas ideas se encaucen en la corriente del pensamiento y la práctica educacional. Por eso, cuando salió el libro de los Caine "Efectuando conexiones» hace 7 años , encargado por la Asociación de Supervisión y Desarrollo de Curriculum y se distribuyó a todos sus miembros, se dieron cuenta que las ideas contenidas en el eran demasiado importantes como para ser ignoradas por los educadores no pertenecientes a la Asociación. Se confeccionó, por tanto, una segunda edición dirigida a todos los educadores en el año 94. Actualmente, las experiencias basadas en el enfoque neurobiológico de la educación se han multiplicado como reguero de pólvora en muchísimas instituciones de América del Norte. De esta manera se estarían cumpliendo en parte los objetivos iniciales propuestos : y es que todos los participantes en un colegio internalizen la teoría del aprendizaje de los Caine de modo que esta se transforme en un modelo mental (Senge 1990) que dirija las decisiones que se deben ir tomando en el proceso de la educación.

Lo que es importante es que el educador tome conciencia de que el cerebro es como cualquier órgano que tiene una función. Su trabajo es aprender. Todo cerebro humano normal tiene una capacidad virtualmente infinita de aprender. Cualquiera sea su edad, sexo, nacionalidad o bagaje cultural. Viene equipado con características excepcionales que, en relación con su aporte al aprendizaje, se resumen en :

- La capacidad para detectar patrones y efectuar aproximaciones ,

- Una capacidad enorme de varios tipos de memoria

- La capacidad de autocorregirse y aprender desde la experiencia por medio del análisis de datos externos y autorreflexión

- Y una infinita capacidad de crear

Si todos tenemos todas estas capacidades, porque nos cuesta tanto educar?

A lo mejor porque todavía no hemos captado cómo aprende el cerebro, especialmente los momentos en que está funcionando de manera óptima. Cuando conozcamos ambas variables, quizás lo podamos hacer mejor.

\section{Características de Funcionamiento Cerebral}

Veamos cuanto se ha avanzado hasta el momento.

Uno de las principales contribuciones de la neurobiología son los aportes de las evidencias sobre el efecto de la experiencia en el aprendizaje La investigaciones sobre el cerebro confirman que las experiencias previas múltiples y complejas son esenciales para que el aprendizaje y la enseñanza sean significativos. Todo evento complejo deja una información en el cerebro la que establece conexiones de lo que se está aprendiendo con el resto de las experiencias del aprendiz, su conocimiento pasado y su conducta futura.

Las evidencias neurobiológicas partieron con experimentos sobre el desarrollo de la 
corteza cerebral de ratas criadas en ambientes enriquecidos ( Diamond, Krech y Rosenzweig 1964; Rosenzweig y Bennet 1977). Encontraron efectos sobre la neocorteza cerebral la que se hacía mas gruesa que la de las ratas criadas en aislamiento o en viveros corrientes. Otros experimentos mostraron que las ramificaciones dendríticas de la corteza visual eran mayores en ratas normales que en ratas deprivadas de la visión (Greenough y Volkmar 1973).

De este modo, el desarrollo del cerebro y el aprendizaje resultan ser como las dos caras de una misma moneda. Las experiencias vitales de una persona literalmente lo llevan a hacer nuevas conexiones entre neuronas y a la secreción de agentes químicos que transmiten las señales. Interpretamos nuevas experiencias e ideas nuevas en base a lo que hemos experimentado o comprendido previamente. Es importante reconocer que tambien existen conexiones no necesariamente aprendidas. Tenemos ciertas capacidades innatas, genéticamente determinadas, tales como el reconocimiento de "arriba" "abajo", "adentro" y "afuera" (Lakoff y Johnson, 1980), sobre las cuales podemos desarrollar nuevas categorías metafóricamente, y tener ideas más complejas como estar "arriba" en una jerarquía o "dentro" de un círculo social.

En suma , la educación basada en el cerebro involucra dos mandatos importantes:

- El diseñar experiencias enriquecedoras y apropiadas parecidas a la vida real de los aprendedores.

- Asegurar que los estudiantes procesen la experiencia de tal manera que aumente la posibilidad de extraer significado.

Tenemos entonces, que la búsqueda de significado tanto por el educador para ensenar como del educando para aprender es un principio importante al que atenerse, y está basado en el conocimiento de que toda experiencia involucra activación y conexión entre neuronas. Este hecho hace más efectivo el aprendizaje nuevo pues conduce a la comprensión más que a la memorización de las materias. Aquí radica la diferencia entre conocimiento superficial y conocimiento con significado.

La búsqueda de significado es innato. Estamos equipados biológicamente para darle un sentido al mundo que nos rodea. Por ejemplo, para Restak (1995) el propósito básico del cerebro es "efectuar representaciones internas de la realidad externa".

La conectividad neuronal y los cambios que experimenta el cerebro por la experiencia se ha denominado Plasticidad Cerebral o plasticidad sináptica (Carlson 1996,p 399), una característica distintiva del cerebro. La analogía del cerebro con una máquina es útil hasta cierto punto, porque es un objeto físico cuyas partes obedecen leyes del mundo material. Por ejemplo, las partes del cerebro se comunican usando medios tales como los neurotransmisores, hormonas, y potenciales de acción. Sin embargo, tambien podemos describirlo como marcadamente plástico y en constante estado de flujo . Cuando describimos fenómenos psicológicos como percepción, aprendizaje y cognición está implícito que estos aspectos de la mente son producto de la máquina física que llamamos cerebro. Esta máquina es diferente de las máquinas que construye el hombre porque está continuamente remodelándose - rearreglando y reordenando las conexiones y relaciones entre las partes, lo que permite que la relación entre los cambios en el comportamiento y los cambios en el cerebro sean bidireccionales.: la experiencia cambia las estructuras neurales, las que a su vez procesan la información entrante de otra forma, lo que da lugar a comportamientos distintos. Las téc- 
nicas de imágenes cerebrales como la tomografía de emisión de positrones (PET) y la Resonancia Magnética Nuclear funcional (fMRI) nos permiten ahora ver los cambios en las respuestas del cerebro que ocurren durante el curso de un experimento de aprendizaje (Jensen, 1998 p2 )

El enriquecimiento ambiental no es la única influencia sobre el cerebro. Tambien lo son la novedad, los desafíos y la retroalimentación (entendida como el conocimiento que obtiene el sujeto del resultado de su acción). El cerebro posee cualidades "elásticas " (Jensen 1998) y está cambiando todo el tiempo. La investigación indica que el cerebro continúa "re-cableándose" con la producción de sinapsis a través de toda la vida. Mientras más se aprende más se generan cambios en el cerebro, y actualmente se sabe que con una estimulación apropiada es capaz de hacer crecer nuevas células, al menos en el hipocampo.

Tambien resulta de interés comprender la relación entre la nutrición y el aprendizaje. La alimentación está constituida por tres agentes químicos-dopamina, serotonina y norepìnefrina- que son los neurotransmisores manufacturados en el cerebro. Reaccionando a la comida que ingerimos, la dopamina y la norepinefrina son agentes químicos de alerta. La serotonina es un agente químico tranquilizador. Cuando se ingiere proteína sola o con carbohidratos aumenta la fuerza cerebral. Por otra parte, cuando se ingiere el carbohidrato solo aumenta la relajación .

El cerebro es un $78 \%$ de agua. Cuando nos deshidratamos hay problemas con la atención, el pensamiento crítico, el aprendizaje y la memoria.

Nuestro cerebro está diseñado para tener altos y bajos de la atención, no una atención continuada. Ya no se pide sostener la atención de los estudiantes y mantenerla. Los momentos en que la atención descansa ayuda al cerebro a fijar las conexiones neurales. Esto conduce a una mejor memoria. Mucho del aprendizaje que se hace en un nivel inconsciente, sin reflexión, permanece en ese nivel. El reloj de nuestro cuerpo parece correr en ciclos de 90-110 minutos. Estos ciclos son los patrones ultradianos, de energía -relajación. Son afectados por nuestra respiración y los niveles de energía afectan tanto nuestro aprendizaje como la percepción de nosotros mismos. Hablando en general, el aprendizaje será mejor en la mañana (10 AM) y temprano en las tardes; el ánimo tiende a ser más pesimista después de almuerzo. La actividad física y el compromiso emocional pueden modificar los ritmos normales del cerebro. Un descanso con ejercicio físico es una excelente forma de alterar un ciclo bajo (Jensen, 1998 ).

Las hormonas, la dieta, las emociones y la química gatillan constantes fluctuaciones en la atención, la memoria y el aprendizaje. El cerebro esta siempre haciendo lo que necesita para sobrevivir, encarar los desafíos y conseguir descanso. Por eso aprendemos mejor con la variedad y la posibilidad de escoger. Como cada uno de nosotros tiene diferentes tablas de tiempo cronológico, biológico y hemisférico, necesitamos espacio para equiparar los tiempos de aprendizaje mas productivo con las tareas a realizar. Según Jensen (p. 65-66), los días de clases conferencias continuadas y directas ya pasaron.

De este modo, el desarrollo del cerebro y el aprendizaje son dos lados de la misma moneda. Las experiencias vitales de una persona literalmente lo llevan a hacer nuevas conexiones entre neuronas y a la secreción de agentes químicos que transmiten las señales. Interpretamos nuevas experiencias e ideas nuevas en base a lo que hemos experimentado o comprendido previamente, lo 
que no necesariamente es aprendido. Tambien existen capacidades innatas, genéticamente determinadas, tales como el reconocimiento de "arriba" "abajo", "adentro" y "afuera" (Lakoff y Johnson, 1980), sobre las cuales podemos desarrollar nuevas categorías metafóricamente, y tener ideas más complejas como estar "arriba" en una jerarquía o "dentro" de un círculo social.

Resumen de principios más importantes sobre el aprendizaje basado en el cerebro:

Los Caine y Crowell (1999) han sintetizado a partir de numerosas investigaciones de diferentes disciplinas algunos principios que pueden servir de base al pensar en el aprendizaje que se produce a través de la educación. Estas disciplinas comprenden campos como las neurociencias, la psicología cognitiva, la teoría del stress y la creatividad. Constituyen principios, que si se analizan con seriedad tendrán como efecto un cambio profundo sobre la educación, concebida hasta hace poco desde una muy diferente perspectiva.

Estos principios son:

1. el cerebro es un sistema viviente: tiene partes con funciones determinadas como los roles del hipocampo en la memoria y las emociones en la amígdala (LeDoux 1996), pero funciona como un todo, con un propósito y una dinámica que permite que la memoria y la emoción se influencien mutuamente.

2. El cerebro/mente es social. El individuo debe ser concebido siempre como parte integrante de un sistema social mas amplio. Esto comienza aun antes del nacimiento. La voz de la madre y su ritmo cardíaco es identificado por el infante. Luego el desarrollo del lenguaje dependerá de escuchar hablar a los demás. Vygotsky (1978) sugiere que incluso la capacidad de tener diálogos internos y pensar es aprendido después de expe- rimentar diálogos externos.

3. La búsqueda de significados es innato. Esta búsqueda está orientada a la supervivencia y puede cambiar con el desarrollo ("Quien soy", "adonde voy") y según Restak (1995) el propósito principal del cerebro es hacer representaciones internas de la realidad. Hasta el bebé , según Gopnik y Meltzoff (1997) nace con la capacidad de desarrollar teorías e hipótesis acerca del funcionamiento del mundo.

4. La búsqueda del significado ocurre a través de patrones: Esto significa el tratar de entender la vida a través de encontrar orden -lo que la ciencia y el arte tienen en común- haciendo categorizaciones, encontrando semejanzas y diferencias y comparando caracteres aislados entre sí. El cerebro puede en forma innata categorizartodo lo redondo, o lo lineal, o lo oscuro, olores básicos., el sentido del número, 'lo que es árbol; hasta las palomas pueden hacer esto. El resultado de todo esto es que el ser humano construye modelos de la realidad. La filosofía del constructivismo en la educación tiene que ver con la creación de tales percepciones y relaciones. La configuración o "configuracionar" esta basado en la fisiología. Grupos de células cerebrales se combinan en redes neuronales que disparan al mismo tiempo en forma consistente. Se requiere del aprendizaje cuando se rompe un patrón o se disrumpe y se necesitan nuevas respuestas. Las nuevas experiencias, significados y comprensiones reconfiguran las configuraciones automáticas. $Y$ a veces estas reconfiguraciones toman tiempo porque los cambios no son puramente mentales sino que fisiológicos.

5. Las emociones son fundamentales para la configuración. El rol de las emociones en el aprendizaje ha sido cambiante .Primero no se consideraron, luego sí pero en forma independiente; ahora se consideran importantes desde las investigaciones de J. 
LeDoux (1996) incluso en el pensamiento de orden superior. Con respecto a lo mismo, Damasio (1994) sostiene que son inseparables y que el cuerpo, el cerebro y las emociones forman una unidad no disociable. La emoción y la cognición interactúan, se energizan y se moldean mutuamente. Restack (1995) añade, "casi todo pensamiento, no importa cuan blando se acompaña de una emoción, no importa cuan sutil". Por eso es tan difícil de cambiar las creencias que tenemos sobre las cosas, porque muchas veces afectan el sentido de lo que somos.

6. Todo cerebro percibe y crea "partes" y "totalidades" en forma simultánea. En último término existen dos tendencias en todos nosotros para organizar la información. Una es reducir la información a partes; la otra es percibir y trabajar con la información como un todo; y esto surge a partir de la organización cerebral.

Las evidencias surgen a partir de los estudios de cerebro dividido que ya hemos mencionado.

\section{El aprendizaje involucra una atención} focalizada a la vez que percepción periférica. La idea central aquí es que el cerebro/mente está inmerso en un mundo de sensaciones, imágenes y recuerdos, y por lo tanto tiene que seleccionar continuamente aquello que atenderá y aquello que debe ignorar. Sin embargo, aunque se esté dirigiendo la atención directamente a algo, el cerebro del niño tambien está absorbiendo información que yace más allá del foco inmediato.

8. El aprendizaje siempre involucra procesos conscientes e inconscientes. Las investigaciones de neurocientistas como Joseph LeDoux están demostrando la realidad del inconsciente. Los procesos del inconsciente cognitivo atraviesan muchos niveles de complejidad mental, los que van desde el análisis rutinario de las características físicas de un estímulo por nuestro sistema sensorial, hasta los recuerdos de eventos pasados, diálogos internos, la recreación de imágenes de objetos no presentes, hasta la toma de decisiones. Gran parte de las cosas que entendemos y adquieren significado son consecuencia de procesamiento inconsciente.

9. Tenemos por lo menos dos formas de organizar la Memoria. Cuando pensamos en la memoria, automáticamente lo relacionamos con lo que está "almacenado" o lo que intentamos "recuperar". Pero no es tan simple como esto, porque la memoria está naturalmente activa todo el tiempo en el momento presente en que nos movemos por el mundo y tratamos de darle sentido a nuestro contexto y nuestras experiencias. Esto es posible gracias a que en nuestro cerebro la organización de las memorias es a la vez estática y dinámica (O’Keefe \& Nadel 1978). La memoria estática es la por todos conocida memoria declarativa (de hechos), la semántica (significados), la procedural (habilidades) y la memoria emocional (los sentimientos). El sistema de memoria dinámica, en cambio, se refiere a un sistema experiencial que registra y organiza los eventos momento a momento de la vida. Las regiones del cerebro importantes para este sistema funcionan en el hipocampo y lo lóbulos prefrontales. Este sistema nos indica donde estamos en el espacio y registra la historia de lo que nos está pasando momento a momento.

10. El aprendizaje es un proceso en desarrollo. La estructura física no solo se desarrolla porque es alimentada y cobijada, sino por las experiencias que tienen las personas las que literalmente llevan a formar nuevas conexiones entre las neuronas y a secretar agentes químicos que transmiten las señales.

La etapa de desarrollo físico mas importante durante el desarrollo son los tres primeros años, pero la plasticidad dura toda la vida. 
La clave más importante para el educador es que todo aprendizaje se construye sobre lo que ha ocurrido anteriormente.

"El mensaje emergente es claro: el cerebro, con su compleja arquitectura e ilimitado potencial es una entidad enormemente plástica y constantemente cambiante, poderosamente modelado por nuestras experiencias de la infancia y a través de toda la vida" M. Diamond \& J. Hopson (1998).

11. El aprendizaje complejo se incrementa por los desafíos se inhibe por las amenazas asociadas con un sentido de desesperanza o fatiga. En el "Cerebro Emocional" (Joseph LeDoux, 1996) muestra como nuestras respuestas de miedo pueden ser explicadas a través de dos sistemas diferentes. Uno que es rápido (talamo-amígdala) y es gatillado si el estímulo es amenzante $y$ otro más lento (que va a la corteza cerebral desde el tálamo), y es consciente.

El camino rápido se puede gatillar por miedo relacionado al sentido de desesperanza o fatiga. En estas circunstancias revertimos a respuestas más primitivas o programadas tempranamente de modo que perdemos el acceso al funcionamiento cerebral superior.

Una alternativa al uso del camino rápido de conexiones es la auto-eficacia, que el niño halla tenido la experiencia de hacer elecciones en un ambiente seguro.

12. Cada cerebro esta organizado en forma única. (como las huellas digitales) Todos tenemos el mismo conjunto de sistemas y organización cerebral; sin embargo, somos todos distintos. Los factores que nos hacen parecidos, son los mismos que a la vez nos hacen distintos Todos nacemos con 100 billones de neuronas. Pero nuestro patrón genético junto con las experiencias particulares de cada uno hace que cada cual tenga un cableado que se diferencia de muchas maneras de cualquier otro.
A pesar de las muchas cosas que tenemos en común, el "input" a nuestros sentidos difiere en cierta medida del de los demás, de todos los que nos han precedido y de todos los que vendrán. La unicidad es un hecho de la vida. La raza, el color, las creencias y la cultura son todos aspectos de la individualidad, pero incluso en culturas muy homogéneas existen inmensas diferencias. La naturaleza es diversa y lucha por esa diversidad, y la sola idea de un solo tipo de flor, de un solo tipo de árbol es tan absurdo como desear que el sistema educacional forme un solo tipo de niño.

De allí que el concepto de las inteligencias múltiples propuesto por Gardner (2000 a), b) ha sido tan bien, recibido por los educadores.

\section{Referencias}

Caine, R.N y Caine, G. ( 1994) Making Connections: Teaching and the Human Brain. AddisonWesley

Caine, G. , Caine, R.N y Crowell, S. (1999) Mind Shifts. (2 $\left.{ }^{\text {nd }} E d.\right)$ Zephyr Press

Carlson, N. R. (1996) Fundamentos de Psicología Fisiológica. $3^{\text {a }}$. Edición Prentice Hall.

Damasio, A. (1994) Descartes' Error: Emotion, Reason and the Human Brain. New York: Avon.

Diamond, M. \& J. Hopson, (1998) Magic Trees of the Mind: How to Nurture Your Child's Intelligence, Creativity and Healthy Emotion. New York: Penguin, Putnam.

Diamond, M. C., KReCh, D. \& Rosenzweig, M.R. (1964) The effect of enriched Environment on the histology of the rat cerebral cortex. Journal of Comparative Neurology, 123, 111-119.

Gardner, H. (2000) The Disciplined Mind. Ed. Penguin books 
GARDNER, H. (2000) Intelligence Reframed. Multiple Intelligences for the $21^{\text {st }}$ century. Basic Books.

Goleman, D. (1995) La Inteligencia Emocional. New York, Bantan.

GreENough, W.T. \& VolKMAR, E.R. (1973) Pattern of dendritic branching in occipital cortex reared in complex environments. Experimental Neurology, 40, 491-504.

Gopnik, A., \& A.N. Meltzoff (1997) Words, Thoughts and Theories.Cambridge, Mass: MIT Press.

JENSEN, E. (1996) Brain Based-Learning. Editorial Turning-Point Publishing

JENSEN, E. (1997) Brain compatible strategies. The Brain Store Inc.

JENSEN, E (1998) Teaching with the Brain in mind. Association for supervision and Curriculum Development.

JENSEN, E. (1998) Introduction to BrainCompatible Learning. The Brain Store.

LAKOFF, E. Y JoHnson, M. (1980) Metaphors we live by. Chicago: Univ. of Chicago Press

LeDoux, J. E. (1996) The Emotional Brain. New York: Simon and Schuster.

RestaK, R. (1995) Brainscapes. New York: Hyperion

Rosenzweig, M.R, KReCH, D., Bennett, E.L. \& Diamond, M. (1962). Effect of environmental complexity and training on brain chemistry and anatomy: A replication and extension. Journal of Comparative and Physiological Psychology, 55, 429-437..

RosenzWeig, M y BeNNetT, E.L. (1977) Effects of environmental enrichment or impoverishment on learning and on brain values in rodents. In A. Oliveno (ed) Genetics, environment, and intelligence (p1-2) Ed. Amsterdam: Elsevier/ North-Holland.

Rosenzweig, M.R., Leiman, A., BreEdlove, S.M. (1999) Biological Psychology. An Introduction to Behavioral, Cognitive and Clinical Neuroscience. Sinauer Assoc. ( $\left.2^{\text {nd }} e d.\right)$

SEnge, P.M. (1990) The Fifth Discipline: the Art and Practice of the Learning Organization. New York: Doubleday Currency.

SPERRY, R.W. (1966) Brain bisection and consciousness. En Brain and Conscious Experience. Ed. J. Eccles. New York: SpringerVerlag.

SPERRY, R.W. (1974). Lateral specialization in the surgically separated hemispheres. In F.O. Schmitt \& F.G. Worden (eds.), Neuroscience $3^{\text {rd }}$ study program . Cambridge: MIT Press.

VyGotsky, L. S. (1978) Mind in Society. Cambridge, Mass: Harvard University Press

Weber, E. (1999) Student Assessment that works. A practical approach. Allyn and Bacon. 